\title{
Mitochondria: enigmatic stewards of the synaptic vesicle reserve pool
}

\author{
Maxim V. Ivannikov' ${ }^{1}$ Kristen M. Harris ${ }^{2}$ and GregoryT. Macleod ${ }^{1 *}$ \\ Department of Physiology, University of Texas Health Science Center at San Antonio, San Antonio, TX, USA \\ 2 Center for Learning and Memory, Section of Neurobiology, Institute for Neuroscience, University of Texas at Austin, Austin, TX, USA \\ *Correspondence: macleod@uthscsa.edu
}

\section{A commentary on}

Synaptic mitochondria in synaptic transmission and organization of vesicle pools in health and disease

by Melissa Vos, Elsa Lauwers and Patrik Verstreken.

Studies on isolated mitochondria and those in cultured cells have revealed much about their inherent biology, and yielded two Nobel prizes, but our knowledge of their role(s) in the onset and progression of neurodegenerative diseases is still limited. In large part, this is because little is known about how mitochondrial biology integrates with neuronal physiology under non-pathological conditions. In this issue of Frontiers in Synaptic Neuroscience, Vos et al. (2010) recognize this gap in our knowledge in their comprehensive review of the role of mitochondria in neurotransmission in health and disease. They bring a novel perspective by considering the mitochondrion's role in the context of functionally defined pools of synaptic vesicles. They conclude that presynaptic mitochondria wield substantial influence on neurotransmission through their ability to mobilize the great majority of synaptic vesicles ( $~ 85 \%$; Rizzoli and Betz, 2005) held in the reserve pool. It is the reserve pool that is called upon to sustain neurotransmitter release during intense neuronal activity approaching the output limits of presynaptic axons. Under pathological conditions, there are conceivably more occasions for operating near output limits, hence even where the primary deficit might not be within the mitochondrion, the limits of mitochondrial function are tested.

In the process of considering which aspects of mitochondrial function are important in neurotransmission the authors open a can of worms which is left in the reader's peripheral vision. The issue is this: different aspects of mitochondrial function are interdependent. This interdependence makes it exceedingly difficult to isolate a particular mitochondrial function for an incisive test of its contribution to neurotransmission. It is this inscrutable quality that has earned the mitochondrion the reputation as an enigmatic player in neurotransmission under both pathological and non-pathological conditions.

Presynaptic mitochondria likely contribute to neurotransmission through a number of aspects of their biology: ATP and neurotransmitter synthesis, the production of reactive oxygen species (ROS), modulation of the local cytosolic $\mathrm{pH}$ and redox state (Giniatullin and Giniatullin, 2003), and regulation of plasma-membrane excitability via $\mathrm{K}_{\text {ATP }}$ channels (Matsumoto et al., 2002). However, it is the ability of mitochondria to take up and release $\mathrm{Ca}^{2+}$ that attracts the most attention - for several reasons. First, the probability of neurotransmitter release has a steep dependence on the concentration of $\mathrm{Ca}^{2+}$ (Brose et al., 1992), enticing many to test for linkages between mitochondrial $\mathrm{Ca}^{2+}$ handling, neurotransmitter release, and calcium-dependent endocytosis. Secondly, $\mathrm{Ca}^{2+}$ is a potent stimulant of mitochondrial respiration, increasing production of both ATP and ROS (Dugan et al., 1995; Jouaville et al., 1999). $\mathrm{Ca}^{2+}$ stimulates mitochondrial production of ATP, which powers the plasma-membrane pumps needed to maintain ion balances essential for $\mathrm{Ca}^{2+}$ homeostasis, thus confounding experiments that seek to elucidate whether it is mitochondrial $\mathrm{Ca}^{2+}$ sequestration or ATP production that is most influential on cytosolic $\mathrm{Ca}^{2+}$ buffering. At the same time, $\mathrm{Ca}^{2+}$-stimulated ROS production makes the mitochondrion a "subject of interest" in studies concerned with activity-dependent acute oxidative stress. Lastly, mitochondria exposed to excessive levels of $\mathrm{Ca}^{2+}$ can "overload" and initiate apoptosis through the release of pro-apoptotic proteins (Kokoszka et al., 2004). Their role as executioner points to mitochondrial dysfunction and the associated $\mathrm{Ca}^{2+}$-dysregulation as possible mechanisms of neurodegeneration.

Advances in optical fluorescence imaging techniques, and the development of synthetic and genetically encoded fluorescent $\mathrm{Ca}^{2+}$-reporters have reinvigorated studies on mitochondrial $\mathrm{Ca}^{2+}$ handling. Despite the advances in imaging techniques, the lack of specific pharmacological tools and information on the genetic identity of mitochondrial proteins (but see Jiang et al., 2009; Perocchi et al., 2010) has hampered progress in elucidating the mechanisms and impact of mitochondrial $\mathrm{Ca}^{2+}$ handling. Meanwhile, data on $\mathrm{Ca}^{2+}$-dependent changes in neuronal ATP levels are essentially absent, except for a small number of studies using luciferase. If we are to understand the relative contributions of mitochondrial $\mathrm{Ca}^{2+}$ sequestration and ATP production to cytosolic $\mathrm{Ca}^{2+}$ buffering and neurotransmission we need a highly dynamic fluorescent ATP or ATP/ ADP reporter. Such reporters are being developed, both synthetic (Hasegawa et al., 2007; Xu et al., 2009) and genetically encoded (Berg et al., 2009; Imamura et al., 2009), but they remain unproven in presynaptic axons. Questions regarding the relative influence of mitochondrial $\mathrm{Ca}^{2+}$ sequestration versus ATP production in $\mathrm{Ca}^{2+}$-buffering and neurotransmission might appear to be quibbling, but the fact remains that it has yet to be shown whether mitochondrial $\mathrm{Ca}^{2+}$ sequestration serves to limit presynaptic $\mathrm{Ca}^{2+}$ levels during axonal firing patterns that are physiological in rate and duration (Chouhan et al., 2010).

On a final note, the experimental systems represented in this review highlight an impediment to our prospects for understanding the role(s) mitochondria play in synaptic transmission in health and disease. Most of the data come from cultured neurons or large experimentally accessible nerve termini (e.g., 


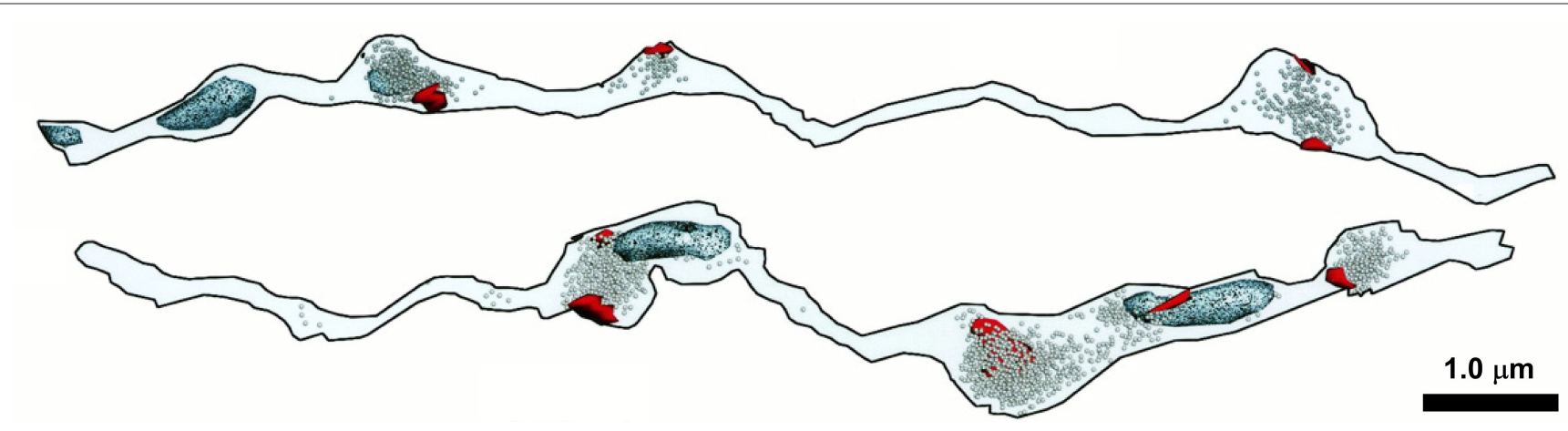

FIGURE 1 |Approximately one-half of synaptic varicosities are devoid of mitochondria in some areas of the mammalian CNS. Ultrastructural features of two 3D reconstructed stratum radiatum axons of area CA1 of the rat hippocampus. Post synaptic densities (red), vesicles (speckled), and mitochondria (light blue) are shown for each axon. Modified and reprinted, with permission, from Shepherd and Harris (1998). See original article for full details. neuromuscular junctions and the Calyx of Held), yet most nerve termini in the mammalian CNS have only a single release site, and only half of them have an attendant mitochondrion (Figure 1; Shepherd and Harris, 1998; Xu-Friedman et al., 2001). Models that recapitulate the architecture of these CNS synapses are required to reveal those mitochondrial roles relevant to neurotransmission in human health and disease. It will be interesting to learn how synaptic boutons without mitochondria derive their ATP to fuel synaptic release, i.e., is it provided by glycolysis or diffusion from a neighboring bouton or perisynaptic astroglia? Are release sites in synaptic boutons without mitochondria competent to release synaptic vesicles? Similarly, it will be fascinating to determine the consequences, if any, for PTP and LTP at synapses that do not possess a presynaptic mitochondrion.

\section{ACKNOWLEDGMENTS}

This work was supported by NIH grants R01 EB002170 and R01 NS021184 to Kristen M. Harris, and R01 NS061914 to Gregory T. Macleod.

\section{REFERENCES}

Berg, J., Hung, Y. P., and Yellen, G. (2009). A genetically encoded fluorescent reporter of ATP:ADP ratio. Nat. Methods 6, 161-166.

Brose, N., Petrenko, A. G., Südhof, T. C., and Jahn, R. (1992). Synaptotagmin: a calcium sensor on the synaptic vesicle surface. Science 256, 1021-1025.
Chouhan, A. K., Zhang, J., Zinsmaier, K. E., and Macleod, G. T. (2010).Presynaptic mitochondria in functionally different motor neurons exhibit similar affinities for $\mathrm{Ca}^{2+}$ but exert little influence as $\mathrm{Ca}^{2+}$ buffers at nerve firing rates in situ. J. Neurosci. 30, 1869-1881.

Dugan, L. L., Sensi, S. L., Canzoniero, L. M., Handran, S. D., Rothman, S. M., Lin, T. S., Goldberg, M. P., and Choi, D. W. (1995). Mitochondrial production of reactive oxygen species in cortical neurons following exposure to $\mathrm{N}$-methyl-D-aspartate. J. Neurosci. 15, 6377-6388.

Giniatullin, A. R., and Giniatullin, R. A. (2003). Dual action of hydrogen peroxide on synaptic transmission at the frog neuromuscular junction. J. Physiol. 552, 283-293.

Hasegawa, T., Hagihara, M., Fukuda, M., and Morii, T. (2007). Stepwise functionalization of ribonucleopeptides: optimization of the response of fluorescent ribonucleopeptide sensors for ATP. Nucleosides Nucleotides Nucleic Acids 26, 1277-1281.

Imamura, H., Nhat, K. P., Togawa, H., Saito, K., Iino, R., Kato-Yamada, Y., Nagai, T., and Noji, H. (2009). Visualization of ATP levels inside single living cells with fluorescence resonance energy transfer-based genetically encoded indicators. Proc. Natl. Acad. Sci. U.S.A. 106, 15651-15656.

Jiang, D., Zhao, L., and Clapham, D. E. (2009). Genomewide RNAi screen identifies Letm 1 as a mitochondrial $\mathrm{Ca}^{2+} / \mathrm{H}^{+}$antiporter. Science 326, 144-147.

Jouaville, L. S., Pinton, P., Bastianutto, C., Rutter, G. A., and Rizzuto, R. (1999). Regulation of mitochondrial ATP synthesis by calcium: evidence for a long-term metabolic priming. Proc. Natl. Acad. Sci. U.S.A. 96, 13807-13812.

Kokoszka, J. E., Waymire, K. G., Levy, S. E., Sligh, J. E., Cai, J., Jones, D. P., MacGregor, G. R., and Wallace, D. C. (2004). The ADP/ATP translocator is not essential for the mitochondrial permeability transition pore. Nature 427, 461-465.

Matsumoto, N., Komiyama, S., and Akaike, N. (2002). Preand postsynaptic ATP-sensitive potassium channels during metabolic inhibition of rat hippocampal CA1 neurons. J. Physiol. 541, 511-520.

Perocchi, F., Gohil, V. M., Girgis, H. S., Bao, X. R., McCombs, J. E., Palmer, A. E., and Mootha, V. K. (2010). MICU1 encodes a mitochondrial EF hand protein required for $\mathrm{Ca}^{2+}$ uptake. Nature. doi:10.1038/ nature09358.

Rizzoli, S. O., and Betz, W. J. (2005). Synaptic vesicle pools. Nat. Rev. Neurosci. 6, 57-69.

Shepherd, G. M., and Harris, K. M. (1998). Threedimensional structure and composition of $\mathrm{CA} 3 \rightarrow \mathrm{CA} 1$ axons in rat hippocampal slices: implications for presynaptic connectivity and compartmentalization. J. Neurosci. 18, 8300-8310.

Vos, M., Lauwers, E., and Verstreken, P. (2010). Synaptic mitochondria in synaptic transmission and organization of vesicle pools in health and disease. Front. Syn. Neurosci. 2, 139. doi:10.3389/ fnsyn.2010.00139.

Xu, Z., Singh, N. J., Lim, J., Pan, J., Kim, H. N., Park, S., Kim, K. S., and Yoon, J. (2009). Unique sandwich stacking of pyrene-adenine-pyrene for selective and ratiometric fluorescent sensing of ATP at physiological pH. J. Am. Chem. Soc. 131, 15528-15533.

Xu-Friedman, M. A., Harris, K. M., and Regehr, W. G. (2001). Three-dimensional comparison of ultrastructural characteristics at depressing and facilitating synapses onto cerebellar Purkinje cells. J. Neurosci. 21, 6666-6672.

Received:09 September 2010; accepted: 10 September 2010; published online: 04 October 2010.

Citation: Ivannikov MV, Harris KM and Macleod GT (2010) Mitochondria: enigmatic stewards of the synaptic vesicle reserve pool. Front. Syn. Neurosci. 2:145. doi: 10.3389/fnsyn.2010.00145

Copyright (C) 2010 Ivannikov, Harris and Macleod. This is an open-access article subject to an exclusive license agreement between the authors and the Frontiers Research Foundation, which permits unrestricted use, distribution, and reproduction in any medium, provided the original authors and source are credited. 